ARTICLE

Received 6 Dec 2012 | Accepted 22 Jan 2013 | Published 26 Feb $2013 \quad$ DOl: 10.1038/ncomms2538

\title{
Directional visible light scattering by silicon nanoparticles
}

Yuan Hsing Fu', Arseniy I. Kuznetsov ${ }^{1}$, Andrey E. Miroshnichenko ${ }^{2}$, Ye Feng $\mathrm{Yu}^{1}$ \& Boris Luk'yanchuk $^{1}$

Directional light scattering by spherical silicon nanoparticles in the visible spectral range is experimentally demonstrated for the first time. These unique optical properties arise because of simultaneous excitation and mutual interference of magnetic and electric dipole resonances inside a single nanosphere. Such behaviour is similar to Kerker's-type scattering by hypothetic magneto-dielectric particles predicted theoretically three decades ago. Here we show that directivity of the far-field radiation pattern of single silicon spheres can be strongly dependent on the light wavelength and the nanoparticle size. For nanoparticles with sizes ranging from 100 to $200 \mathrm{~nm}$, forward-to-backward scattering ratio above six can be experimentally obtained, making them similar to 'Huygens' sources. Unique optical properties of silicon nanoparticles make them promising for design of novel low-loss visible- and telecom-range metamaterials and nanoantenna devices.

\footnotetext{
${ }^{1}$ Data Storage Institute, Advanced Concepts and Nanotechnology Division, 5 Engineering Drive 1, Singapore 117608, Singapore. ${ }^{2}$ Nonlinear Physics Centre, Research School of Physics and Engineering, The Australian National University, 59 Mills Road, Canberra ACT 0200, Australian Capital Territory, Australia. Correspondence and requests for materials should be addressed to A.I.K. (email: arseniy_k@dsi.a-star.edu.sg) or Y.H.F.

(email: FU_Yuan_Hsing@dsi.a-star.edu.sg).
} 
E fficient control of the visible light at the nanoscale dimensions is important for future light-on-chip integration. At the subwavelength scale conventional optical elements, such as lenses, are not functional, which requires conceptually new types of nanoscale optical devices. Resonant plasmonic structures are one of the most promising solutions to this problem due to their ability to capture and concentrate visible light at subwavelength dimensions ${ }^{1,2}$. Different types of nanoscale optical devices such as nanolenses ${ }^{3,4}$, nano-waveguides ${ }^{5,6}$, nanoantennas ${ }^{7-14}$ and so on based on metallic nanoparticles have been demonstrated. Many of these functional devices such as nanoantennas are designed in analogy with microwave optics, where metals are routinely used for manipulation of the electromagnetic radiation $8,9,12$. The main drawback of using plasmonic particles in the visible frequency range is their intrinsic losses, which strongly affect their overall performance and limits their scalability to dimensions for practical use. One of the possible ways to avoid such limitation and still have similar resonant properties is to use high-refractive index dielectric nanoparticles ${ }^{15,16}$. Recently, visible-range nanoantennas based on spherical silicon nanoparticles have been theoretically analysed demonstrating higher efficiency compared with their metallic analogues ${ }^{17-19}$. However, experimental demonstrations of such antennas have been limited to millimetre scales and microwave frequencies ${ }^{20}$.

According to theoretical predictions based on Mie theory highrefractive index dielectric nanoparticles of spherical shape can have strong dipole-like resonances in the visible spectral range ${ }^{21,22}$. In contrast to spherical metallic nanoparticles, for which the resonant scattering is dominated by the electric-type resonances (electric dipole, quadrupole and so on $)^{23}$, dielectric nanoparticles can have both electric and magnetic dipole resonances simultaneously excited inside the same particle ${ }^{21,22,24}$. The magnetic dipole response of dielectric particles originates from the circular displacement currents excited inside the particle by incident light. Recently, first experimental demonstrations of a very-strong magnetic dipole resonance in the visible spectral range using silicon nanoparticles have been published ${ }^{25,26}$.

On the other hand, as shown in recent theoretical works, interference of magnetic and electric dipole resonances inside high-refractive index dielectric nanoparticles can strongly affect their scattering pattern making it dependent on wavelength ${ }^{27,28}$. For some wavelengths the particles can act as 'Huygens' sources, scattering the whole energy in the forward direction, while for another wavelength range, light is almost completely scattered backward. This behaviour is similar to light scattering by hypothetic magneto-dielectric particles theoretically predicted by Kerker et al. ${ }^{29}$ in 1983. Similar results were also discussed for purely dielectric spherical particles by Videen and Bickel ${ }^{30}$ in 1992. Such behaviour is also associated with the directional Fano resonance ${ }^{31-33}$, when the scattering is strongly enhanced because of constructive resonant interference in one direction and suppressed in the opposite one. It can be achieved for example, in the system with two dipole-like excitations, one of them being at the resonance ${ }^{34}$.

Very recently first experimental demonstration of Kerker'stype scattering by high-refractive index dielectric particles has been published ${ }^{35}$. These results were obtained in $\mathrm{GHz}$ frequency range with millimetre-size particles. The authors claimed scalability of this interesting electromagnetic problem for all frequency ranges. However, to date no experimental proof of this concept in the optical spectral range has been demonstrated. Realization of such relatively simple nano-optical systems having both electric and magnetic dipole resonances with shifted phases would allow scaling the optical nanoantenna concept down to a single nanoparticle, which can redirect incoming light in different directions depending on wavelength and size ${ }^{17,18,36,37}$.
In this paper, we present the first experimental study of directional scattering by spherical silicon nanoparticles in the visible spectral range. We demonstrate strong anisotropy of nanoparticle scattering in forward and backward directions. This leads to the appearance of a large spectral range where forward scattering strongly dominates, exceeding its backward counterpart by more than six times.

\section{Results}

Theoretical modelling using Mie theory. Figure 1 shows scattering properties of a spherical silicon nanoparticle with radius of $75 \mathrm{~nm}$ in free space calculated by Mie theory ${ }^{38}$. The particle is excited by a plane wave from top and the scattering into the upper (backward) or lower (forward) hemispheres are calculated as shown in the inset to Fig. 1a. Figure 1a represents forward (green curve) and backward (blue curve) scattering spectra together with the forward-to-backward ratio calculated dividing one spectrum by another (orange curve). The scattering spectra into forward and backward directions are very different. Both spectra have two well defined maxima in the visible spectral range, which can be attributed to electric (around $500 \mathrm{~nm}$ ) and magnetic (around $600 \mathrm{~nm}$ ) dipole resonances ${ }^{25}$. However, one can see a noticeable shift of the directional scattering resonance positions. For backward scattering, the electric and magnetic dipole resonances are located closer to each other than in the case of forward scattering. There are three well defined spectral ranges with different scattering properties. In the first spectral range with wavelengths $\lambda>603 \mathrm{~nm}$, forward scattering dominates while
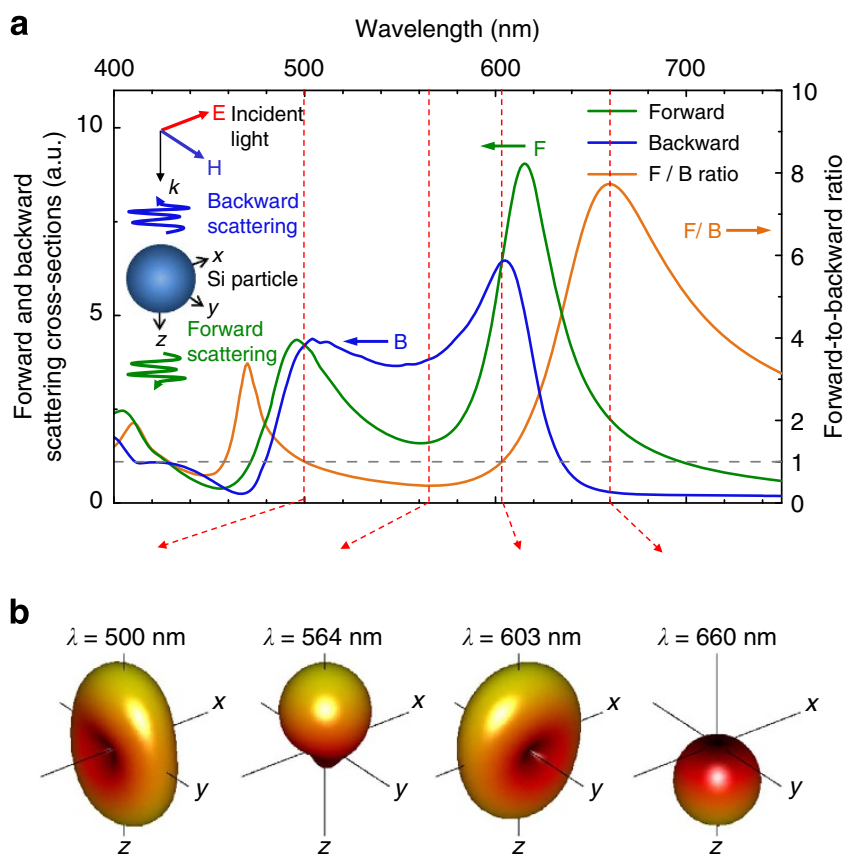

Figure 1 | Scattering properties of a silicon (Si) nanoparticle with radius of $\mathbf{7 5} \mathbf{~ n m}$ in free space calculated by Mie theory. (a) Forward (green curve) and the backward (blue curve) scattering cross-sections, and the forward-to-backward ratio (orange curve) of the Si nanoparticle. The grey dashed line represents the values of forward-to-backward ratio equal to unity. Inset into $\mathbf{a}$ is the calculation scheme: the nanoparticle is excited by a plane wave from top while the scattered light is integrated over the upper and lower hemispheres for backward and forward scattering. (b) Far-field scattering radiation patterns in the four spectral points: $660 \mathrm{~nm}$-maximum forward-to-backward ratio; 564 nm-minimum forward-to-backward ratio; 500 and $603 \mathrm{~nm}$-equal forward and backward scattering at electric $(500 \mathrm{~nm})$ and magnetic $(603 \mathrm{~nm})$ dipole resonances. 
a

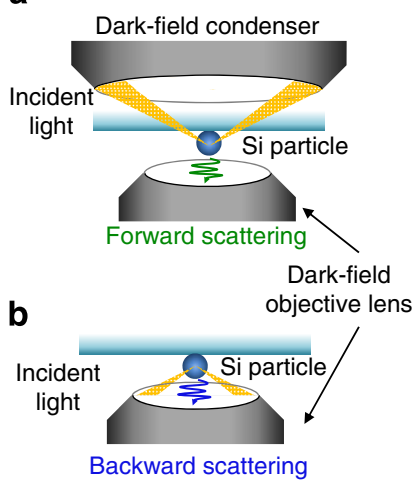

C

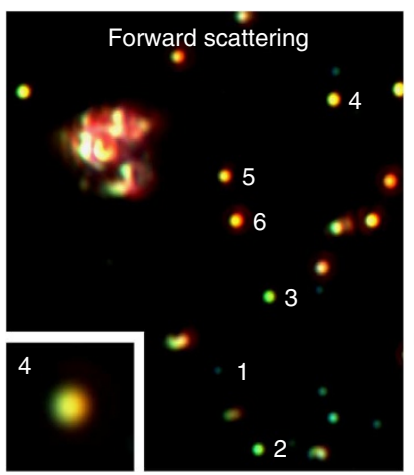

d

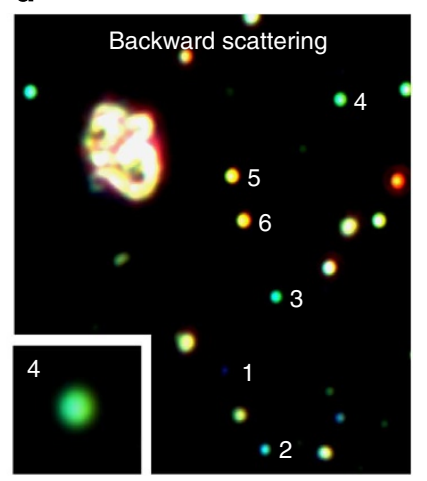

Figure 2 | Transmission and reflection dark-field microscope images of laser-generated Si nanoparticles. (a,b) Schemes of forward and backward scattering measurements. (c,d) True colour CCD (charge-coupled device) images of the forward and backward scattering by Si nanoparticles. Selected nanoparticles are marked by corresponding numbers from 1 to 6 in both images. Inset into (c,d) shows magnified dark-field microscope image of nanoparticle \#4 in forward and backward scattering directions.

backward scattering is almost zero, which gives rise to the forwardto-backward ratio reaching a maximum value of $\sim 8$ at $660 \mathrm{~nm}$. The calculated angular scattering diagram at this wavelength is plotted in Fig. $1 \mathrm{~b}$ and demonstrates almost perfect alignment of the scattering into the forward direction. In the second wavelength range of $500 \mathrm{~nm}<\lambda<603 \mathrm{~nm}$ the situation completely changes. The backward scattering is dominant while forward-to-backward ratio goes below 0.5 at $564 \mathrm{~nm}$. The scattering diagram at this wavelength (also shown in Fig. 1b) demonstrates the scattering alignment into the backward direction with minor residual scattering in the forward direction. In the third spectral range $\lambda<500 \mathrm{~nm}$ the forward-to-backward ratio starts to grow again, which is accompanied by a reduction of total scattering making this spectral range less attractive. Two more interesting spectral points can be noted at 603 and $500 \mathrm{~nm}$ where the forward and backward scattering intensities are equal. At $603 \mathrm{~nm}$ the scattering pattern corresponds to a magnetic dipole aligned in the $\mathrm{Y}$ direction parallel to the magnetic field of incoming light, while at $500 \mathrm{~nm}$ it corresponds to an electric dipole aligned in the $\mathrm{X}$ direction parallel to the electric field (Fig. 1b).

Scattering anisotropy described above has recently been discussed theoretically for silicon ${ }^{27}$ and germanium ${ }^{28}$ nanoparticles. In particular, it was shown that the wavelengths of the maximum forward and backward scattering correspond well to conditions derived by Kerker et al. for hypothetic magnetodielectric particles ${ }^{29}$. The reason for anisotropic scattering is the presence of two orthogonal dipole resonances (electric and magnetic), whose interference influences the total scattering pattern. The remainder of this paper will concentrate on experimental demonstration of this concept in the visible spectral range.

Microscopic analysis. In this paper, we used femtosecond laser ablation method to produce silicon nanoparticles of various sizes. In contrast to the previous studies ${ }^{25}$, the ablated silicon wafer was covered by an additional glass substrate. The ablated nanoparticles were collected and solidified on this glass substrate forming silicon nanoparticles of almost perfect spherical shape. This method for particle generation is somewhat similar to laser-induced transfer ${ }^{39-42}$, but with no real control over nanoparticle size and position on the substrate. Figure 2 shows dark-field microscope images of silicon nanoparticles collected on the glass substrate. Both reflected and transmitted dark-field images have been recorded, showing scattering by these nanoparticles in backward and in forward directions (see experimental geometries in Fig. 2a,b and Methods section for details). In these images, fabricated nanoparticles shine by all the rainbow colours, which correspond to their strong magnetic and electric dipole scattering ${ }^{25}$. However, the colours of the same particles are systematically different in the forward and in the backward directions (see side-to-side comparison in Fig. 2c,d). For example, particle \#2 has a blue colour in the backward scattering direction, and is green in the forward scattering direction. The same is for particle \#4, which changes colour from green (backward) to yellow (forward). Similar colour modifications can be observed for each silicon nanoparticle in Fig. 2. Such colour changes in forward and backward directions have earlier been observed in gold-silver dimers exhibiting Fanotype resonances ${ }^{34}$. In the case of metallic dimer, two electric dipoles excited in different nanoparticles interfere providing Fano-type resonant anisotropic scattering. In silicon nanoparticles, both electric and magnetic dipoles are excited simultaneously, which gives rise to anisotropic scattering by a single nanoparticle of spherical shape.

Spectroscopic analysis. To account for this effect, we performed single nanoparticle spectroscopy measurements for each of six nanoparticles marked in Fig. 2 with numbers 1-6 in both forward and backward scattering directions (see details in Methods section). Results of these measurements are shown in Fig. 3 together with the nanoparticle dark-field microscope and scanning electron microscopy (SEM) images. As it can be seen from the SEM analysis each shiny object in Fig. 2 corresponds to a silicon nanoparticle of shape close to spherical and diameter ranging from 100 to $200 \mathrm{~nm}$. However, forward (green curves) and backward (blue curves) scattering spectra are very different from each other for each nanoparticle. The resonance maxima positions in the backward scattering are much closer one to each other, which is similar to theoretically predicted behaviour, making them almost indistinguishable in the experiment. On the other hand, maxima in the forward scattering spectra are well defined. This provides a large spectral range at the long-wavelength side where forward scattering is strongly dominant over backward. Orange curves in Fig. 3 show experimental data for the forward-to-backward ratio, which was obtained for each nanoparticle dividing one scattering spectrum by another. This ratio has well-defined maximum at the long-wavelength spectral side and reaches value above six for some nanoparticle sizes (see the right axes in Fig. 3). In this spectral range, silicon nanoparticles act as 'Huygens' sources scattering light only in forward direction. On the other hand, in the central spectral range between electric 


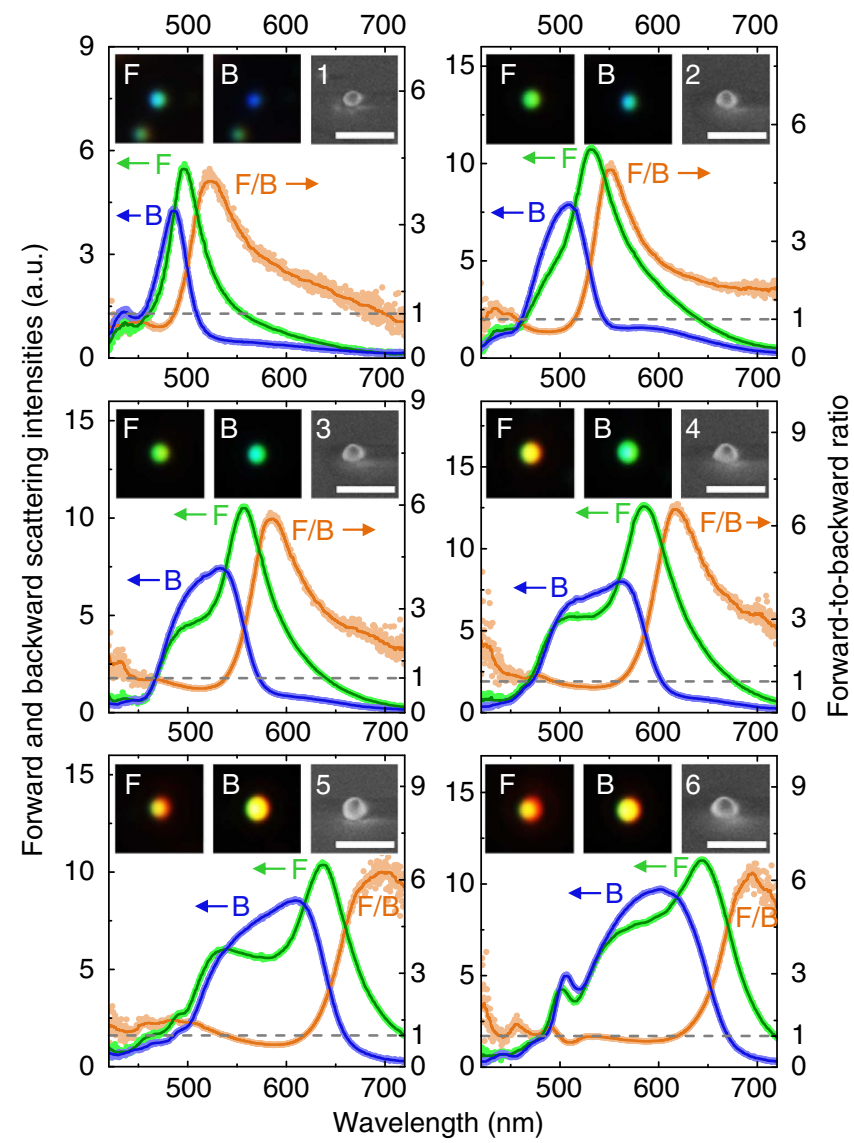

Figure 3 | Experimentally measured forward and backward scattering spectra. Left axes show forward (green) and backward (blue) scattering intensities, and right axes show forward-to-backward ratio (orange curves) of silicon nanoparticles selected in Fig. 2. Dashed lines represent the forward-to-backward ratio equal to unity. Dots represent experimental data, and solid lines are their computer-generated smoothing. Insets show close view transmitted (F) and reflected (B) dark-field microscope images, and SEM images taken at an angle of $52^{\circ}$ for each selected nanoparticle (1-6). The scale bar in the SEM images is $500 \mathrm{~nm}$.

and magnetic dipole resonances the backward scattering becomes dominant. Experimentally measured spectral behaviour is very similar to the theoretically predicted one and proves that a single silicon nanoparticle can controllably redirect incoming light in forward or backward direction depending on nanoparticle size and irradiation wavelength.

\section{Discussion}

Experimentally observed spectra are quite similar to theoretical calculations shown in Fig. 1 (see particles \#4 and 5 for close resonance positions). However, one can also see some differences. Electric dipole resonances in the experiment are less pronounced and located closer to magnetic dipole resonance peaks than in the theory, which makes them almost indistinguishable in the backward scattering measurements. We hypothesize this difference arises from non-ideality of the nanoparticle shape. The SEM analysis (see insets in Fig. 3) shows that almost all nanoparticles are slightly squeezed in vertical direction. To account for this effect, we calculated spectral properties of nanoparticles with spheroidal shape with $\mathrm{X}$ and $\mathrm{Y}$ radii $\left(R_{\mathrm{x}}\right.$ and $\left.R_{\mathrm{y}}\right)$ fixed at $75 \mathrm{~nm}$ and radius in $\mathrm{Z}$ direction $\left(R_{\mathrm{z}}\right)$ varied from 75 to $40 \mathrm{~nm}$ (see Fig. 4). These calculations were performed using finitedifference time-domain method by commercial finite-difference

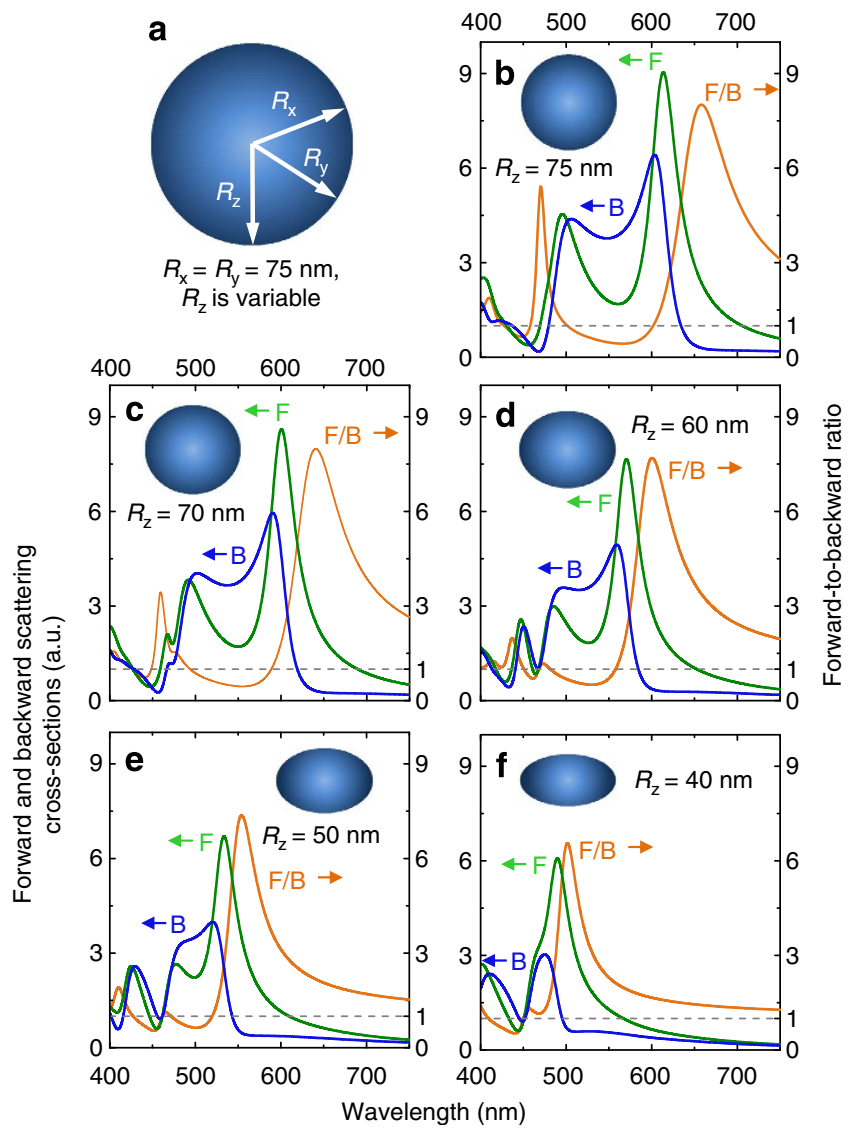

Figure 4 | Influence of the shape of Si nanoparticles on their spectral properties. (a) Schematic representation of a spheroidal nanoparticle with $R_{\mathrm{x}}=R_{\mathrm{y}}$, and varied $R_{\mathrm{z}} . R_{\mathrm{x}}$ and $R_{\mathrm{y}}$ are fixed at $75 \mathrm{~nm}$. (b-f) Calculated results of forward (green curves) and backward (blue curves) scattering, and forward-to-backward ratio (orange curves) for silicon nanoparticles with $R_{\mathrm{z}}=75,70,60,50$ and $40 \mathrm{~nm}$, respectively. Calculations are done in free space using finite-difference time-domain method.

time-domain software (Lumerical Solutions Inc.) in the same geometry as in Fig. 1. When the particle is squeezed in $\mathrm{Z}$ direction the scattering electric and magnetic resonances become less pronounced and shift closer to each other. This leads to overlapping of the resonances in the backward scattering at relatively large aspect ratios $\left(R_{\mathrm{z}}<60 \mathrm{~nm}\right)$. This spectral behaviour is similar to that experimentally observed for particle \#4, which has slightly spheroidal shape (see Fig. 3 (\#4) and Fig. 4d for direct comparison). On the other hand, particle $\# 5$ has a shape much closer to a sphere, which results in better defined electric and magnetic dipole resonances in both forward and backward scattering directions. Another interesting feature arising from these calculations is that the maximum of the forward-tobackward ratio shifts to shorter wavelengths and approaches the maximum of forward scattering when the particle is squeezed in $\mathrm{Z}$ direction. This results from spectral overlap of electric and magnetic dipole resonances and leads to an increase of the total scattering in the forward direction. Similar effects were recently theoretically predicted for silver core/silicon shell nanoparticles where overlapping of electric and magnetic resonance was achieved by changing the size of the silver core ${ }^{19}$.

Further deviations between theory and experiment arise because of the glass substrate, which effect shifts electric dipole resonance to the longer wavelengths closer to magnetic dipole resonance due to 'dressing effect' ${ }^{25}$. This behaviour is in 
a

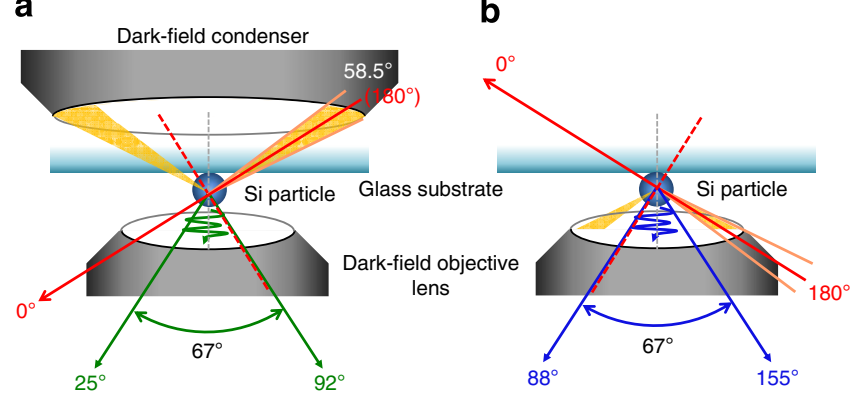

Figure 5 | Detailed schematic illustrations of the forward and backward scattering measurements. According to these schemes the (a) forward and (b) backward scatterings were measured separately.

good correlation with our experimental results (Fig. 3). We should also note that small difference between experimental nanoparticle sizes obtained from SEM analysis and those predicted theoretically for the same resonance positions can be explained by the presence of a thin natural oxide layer on top of the nanoparticles, which effectively decreases the silicon nanoparticle radius ${ }^{26}$.

In spite of discussed limitations of the applicability of the Mie theory to the real experimental situation, one can see a very good correspondence between experimental and theoretical shapes of the forward and backward scattering spectra. The main conclusions derived by Mie theory related to the presence of spectral regions with suppressed backward or forward scattering by a single dielectric particle are clearly demonstrated in our experiments in the visible spectral range. It is along the line of the Kerker's-type scattering predicted in 1983 (ref. 29).

In conclusion, it is experimentally demonstrated for the first time that silicon nanoparticles of spherical shape exhibit strongly anisotropic scattering in the visible spectral range. This unique scattering behaviour results from interference of electric and magnetic dipoles, excited by external illumination. As a result in a broad spectral range these nanoparticles can scatter the most of energy in the forward direction acting like 'Huygens' sources. For a different spectral range the most of the energy is scattered in the backward direction. Control and fine tuning of these spectral regions is possible because of almost linear dependence of the scattering resonances on the nanoparticle size. It is also shown that by squeezing the nanoparticles in the direction of light propagation, it is possible to further overlap electric and magnetic dipole resonances. This results in a noticeable increase of their forward scattering.

These unique optical properties and very low losses compared with plasmonic nanoparticles make silicon nanoparticles perfect candidates for design of high-performance nanoantennas, lowloss metamaterials and other novel nanophotonic devices.

After submission of this paper a preprint of another related manuscript appeared at ArXiv database ${ }^{43}$. In this work, authors experimentally demonstrated Kerker's-type zero backward scattering by GaAs pillars in the red part of visible spectrum.

\section{Methods}

Si nanoparticles of various sizes were fabricated by femtosecond laser ablation of a silicon wafer. A glass substrate was placed on top of the wafer. The laser beam passed through the glass substrate and ablated silicon surface. Ablated silicon nanoparticles were collected and solidified on the glass substrate. Then they were studied by single nanoparticle dark-field spectroscopy, dark-field optical microscopy and scanning electron microscopy (DA300, FEI). In the laser experiments, we used a commercial $1 \mathrm{kHz}$ femtosecond laser system (Tsunami + Spitfire, Spectra Physics) delivering $1 \mathrm{~mJ}, 100 \mathrm{fs}$ laser pulses at a central wavelength of $800 \mathrm{~nm}$. The laser beam with diameter of $4 \mathrm{~mm}$ was focused onto the sample surface by a $\times 20$ microscope objective (Mitutoyo, MPlan NIR 20). Laser irradiation conditions were chosen to ablate a moderate amount of nanoparticles, high enough to have all the required sizes but small enough to keep individual nanoparticles separated from each other ${ }^{25}$. The laser beam was scanned through the sample surface at average power of $0.25 \mathrm{~mW}$ and scanning speed of $1 \mathrm{~mm} \mathrm{~s}^{-1}$. These parameters are slightly above the ablation threshold of silicon at these irradiation conditions. Scanned lines were separated by a pitch of $100 \mu \mathrm{m}$ to avoid overlapping of ablated particles from different scans. The ablated particles were observed as bright coloured spots in a dark-field microscope (Nikon, Ti-U) at $\times 50$ magnification.

Optical scattering properties of single nanoparticles were studied with an optical dark-field microscope (Nikon, Ti-U) equipped with a high-sensitivity spectrometer (Andor SR-303i) and a $400 \times 1,600$ pixel EMCCD (Andor Newton) described elsewhere ${ }^{25}$. Figure 5 shows how the forward and backward scattering of single nanoparticles were measured. Si nanoparticles on glass substrate were placed facedown onto the sample holder of the microscope. During forward scattering measurements, the light from the upper dark-field condenser shone onto the sample surface (orange lines), and the light scattered in the forward direction (green arrows) was collected by the lower dark-field objective lens.

During backward scattering measurements, the light shone from the lower darkfield objective lens (orange lines), and the light scattered in the backward direction (blue arrows) was collected by the same lens. The numerical aperture of the upper dark-field condenser and the lower dark-field objective lens for light incidence was $0.8-0.9$. This provided the angle of light incidence onto the nanoparticle sample in the range from $53^{\circ}$ to $64^{\circ}$ with average value of $58.5^{\circ}$ with respect to the normal to the surface. The numerical aperture of the bottom objective lens for light collection was of 0.55 , which led to the total angle for light collection of $67^{\circ}$. Considering the light propagation directions along the red lines, which are marked by signs $0^{\circ}$ and $180^{\circ}$ in Fig. 5a,b, the collection angle for light scattering was from $25^{\circ}$ to $92^{\circ}$ in (a) and from $88^{\circ}$ to $155^{\circ}$ in (b) relative to these directions. Taking into account rotational symmetry this corresponded to about a half of a total forward particle scattering in (a) and a half of a total backward particle scattering in (b). No backward scattering was collected in these conditions in (a) and no forward scattering in (b). As a result, the forward and backward scatterings were measured separately. To normalize forward and backward scattering intensities to the same level, scattering by a subwavelength dust spot was measured in both geometries and used for normalization. A coloured camera was also attached to another port of the microscope to collect optical images of the scattering pattern in the dark-field illumination mode. The scattering spectra were measured over the wavelength range of $400-800 \mathrm{~nm}$.

\section{References}

1. Novotny, L. \& Hecht, B. Princples of Nano-Optics (Cambridge University Press, 2006).

2. Kawata, S., Ohtsu, M. \& Irie, M. Nano-Optics (Springer-Verlag, 2002).

3. Li, K., Stockman, M. I. \& Bergman, D. J. Self-similar chain of metal nanospheres as an efficient nanolens. Phys. Rev. Lett. 91, 227402 (2003).

4. Dai, J., Cajko, F., Tsukerman, I. \& Stockman, M. I. Electrodynamic effects in plasmonic nanolenses. Phys. Rev. B 77, 115419 (2008).

5. Brongersma, M. L., Hartman, J. W. \& Atwater, H. A. Electromagnetic energy transfer and switching in nanoparticle chain arrays below the diffraction limit. Phys. Rev. B 62, R16356-R16359 (2000).

6. Maier, S. A. et al. Local detection of electromagnetic energy transport below the diffraction limit in metal nanoparticle plasmon waveguides. Nature Mater. 2, 229-232 (2003).

7. Bharadwaj, P., Deutsch, B. \& Novotny, L. Optical antennas. Adv. Opt. Photon 1, 438-483 (2009).

8. Curto, A. G. et al. Unidirectional emission of a quantum dot coupled to a nanoantenna. Science 329, 930-933 (2010).

9. Dregely, D. et al. 3D optical Yagi-Uda nanoantenna array. Nature Comm. 2, 267 (2011).

10. Novotny, L. \& van Hulst, N. F. Antennas for light. Nature Photon. 5, 83-90 (2011).

11. Giannini, V., Fernandez-Dominguez, A. I., Heck, S. C. \& Maier, S. A. Plasmonic nanoantennas: fundamentals and their use in controlling the radiative properties of nanoemitters. Chem. Rev. 111, 3888-3912 (2011).

12. Maksymov, I. S., Staude, I., Miroshnichenko, A. E. \& Kivshar, Y. S. Optical Yagi-Uda nanoantennas. Nanophotonics 1, 65-81 (2012).

13. Mirin, N. A. \& Halas, N. J. Light-bending nanoparticles. Nano Lett. 9, 1255-1259 (2009).

14. King, N. S. et al. Angle- and spectral-dependent light scattering from plasmonic nanocups. ACS Nano. 5, 7254-7262 (2011).

15. Zhao, Q., Zhou, J., Zhang, F. \& Lippens, D. Mie resonance-based dielectric metamaterials. Mater. Today 12, 60-69 (2009).

16. Soukoulis, C. M. \& Wegener, M. Past achievements and future challenges in the development of three-dimensional photonic metamaterials. Nature Photon. 5, 523-530 (2011).

17. Krasnok, A. E., Miroshnichenko, A. E., Belov, P. A. \& Kivshar, Y. S. Huygens optical elements and Yagi-Uda nanoantennas based on dielectric nanoparticles. JETP Lett. 94, 593-598 (2011). 
18. Krasnok, A. E., Miroshnichenko, A. E., Belov, P. A. \& Kivshar, Y. S. All-dielectric optical nanoantennas. Opt. Express 20, 20599-20604 (2012).

19. Liu, W., Miroshnichenko, A. E., Neshev, D. N. \& Kivshar, Y. S. Broadband unidirectional scattering by magneto-electric core-shell nanoparticles. ACS Nano. 6, 5489-5497 (2012).

20. Filonov, D. S. et al. Experimental verification of the concept of all-dielectric nanoantennas. Appl. Phys. Lett. 100, 201113 (2012).

21. Evlyukhin, A. B., Reinhardt, C., Seidel, A., Luk'yanchuk, B. S. \& Chichkov, B. N. Optical response features of Si-nanoparticle arrays. Phys. Rev. B 82, 045404 (2010).

22. Garcia-Etxarri, A. et al. Strong magnetic response of submicron silicon particles in the infrared. Opt. Express 19, 4815-4826 (2011).

23. Evlyukhin, A. B. et al. Optical properties of spherical gold mesoparticles. Appl. Phys. B 106, 841-848 (2012).

24. Evlyukhin, A. B., Reinhardt, C. \& Chichkov, B. N. Multipole light scattering by nonspherical nanoparticles in the discrete dipole approximation. Phys. Rev. B 84, 235429 (2011).

25. Kuznetsov, A. I., Miroshnichenko, A. E., Fu, Y. H., Zhang, J. B. \& Luk'yanchuk, B. Magnetic light. Sci. Rep. 2, 492 (2012).

26. Evlyukhin, A. B. et al. Demonstration of magnetic dipole resonances of dielectric nanospheres in the visible region. Nano Lett. 12, 3749-3755 (2012).

27. Nieto-Vesperinas, M., Gomez-Medina, R. \& Saenz, J. J. Angle-suppressed scattering and optical forces on submicrometer dielectric particles. J. Opt. Soc. Am. A 28, 54-60 (2011)

28. Gomez-Medina, R. et al. Electric and magnetic dipolar response of germanium nanospheres: interference effects, scattering anisotropy, and optical forces. J. Nanophoton. 5, 053512 (2011).

29. Kerker, M., Wang, D. \& Giles, G. Electromagnetic scattering by magnetic spheres. J. Opt. Soc. Am. 73, 765-767 (1983).

30. Videen, G. \& Bickel, W. S. Light scattering resonances in small spheres. Phys. Rev. A 45, 6008-6012 (1992).

31. Luk'yanchuk, B. et al. The Fano resonance in plasmonic nanostructures and metamaterials. Nature Mater. 9, 707-715 (2010).

32. Miroshnichenko, A. E., Flach, S. \& Kivshar, Y. S. Fano resonances in nanoscale structures. Rev. Mod. Phys. 82, 2257-2298 (2010).

33. Rahmani, M., Luk'yanchuk, B. \& Hong, M. Fano resonance in novel plasmonic nanostructures. Las. Photon. Rev. doi:10.1002/lpor.201200021 (2012).

34. Shegai, T., Chen, S., Miljković, V. D., Zengin, G., Johansson, P. \& Käll, M. A bimetallic nanoantenna for directional colour routing. Nature Comm. 2, 481 (2011).

35. Geffrin, J. M. et al. Magnetic and electric coherence in forward- and backscattered electromagnetic waves by a single dielectric subwavelength sphere. Nature Comm. 3, 1171 (2012).

36. Rolly, B., Stout, B. \& Bonod, N. Boosting the directivity of optical antennas with magnetic and electric dipolar resonant particles. Opt. Expr. 20, 20376-20386 (2012)
37. Schmidt, M. K. et al. Dielectric antennas-a suitable platform for controlling magnetic dipolar emission. Opt. Expr. 20, 13636-13650 (2012).

38. Bohren, C. F. \& Huffman, D. R. Absorption and Scattering of Light by Small Particles (Wiley, 1983)

39. Kuznetsov, A. I., Koch, J. \& Chichkov, B. N. Laser-induced backward transfer of gold nanodroplets. Opt. Expr. 17, 18820-18825 (2009).

40. Kuznetsov, A. I. et al. Laser-induced transfer of metallic nanodroplets for plasmonics and metamaterial applications. J. Opt. Soc. Am. B 26, B130-B138 (2009).

41. Kuznetsov, A. I., Kiyan, R. \& Chichkov, B. N. Laser fabrication of 2D and 3D microstructures from metal nanoparticles. Opt. Expr. 18, 21198-21203 (2010)

42. Kuznetsov, A. I. et al. Laser fabrication of large-scale nanoparticle arrays for sensing applications. ACS Nano. 5, 4843-4849 (2011).

43. Person, S. et al. Demonstration of zero optical backscattering from single nanoparticles. Preprint at http://arxiv.org/abs/1212.2793v1 (2012)

\section{Acknowledgements}

We thank Ms Janaki DO Shanmugam (DSI) for help with SEM analysis and Reuben Backer (DSI) for editing. This work was supported by the Agency for Science, Technology and Research $\left(A^{\star}\right.$ STAR) of Singapore: SERC Metamaterials Programme on Superlens, grant no. 092154 0099; SERC grant no. TSRP-102 152 0018; the grant no. JCOAG03-FG04-2009 from the Joint Council of A*STAR, and by the Australian Research Council through the Future Fellowship project FT110100037.

\section{Author contributions}

Y.H.F., A.I.K., and B.L. contributed to initial idea generation; Y.H.F. performed single nanoparticle spectroscopy and FDTD modelling, contributed to SEM analysis and wrote the first paper draft; A.I.K. fabricated Si nanoparticles, contributed to single nanoparticles spectroscopy and SEM analysis, wrote the final manuscript and coordinated the whole work; A.E.M. performed Mie theory simulations of a Si nanoparticle in free space and theoretical analysis of a Si nanoparticle on a substrate; Y.F.Y. contributed to discussions and FDTD simulations; B.L. and A.E.M. contributed to the manuscript preparation. All authors read and corrected the manuscript before the submission.

\section{Additional information}

Competing financial interests: The authors declare no competing financial interests.

Reprints and permission information is available online at http://npg.nature.com/ reprintsandpermissions/

How to cite this article: Fu, Y. H. et al. Directional visible light scattering by silicon nanoparticles. Nat. Commun. 4:1527 doi: 10.1038/ncomms2538 (2013). 